\title{
Strong Law of Large Numbers under an Upper Probability
}

\author{
Xiaoyan Chen \\ Graduate Department of Financial Engineering, Ajou University, Suwon, South Korea \\ Email: cxy_161977@163.com
}

Received September 3, 2012; revised October 3, 2012; accepted October 10, 2012

\begin{abstract}
Strong law of large numbers is a fundamental theory in probability and statistics. When the measure tool is nonadditive, this law is very different from additive case. In 2010 Chen investigated the strong law of large numbers under upper probability $V$ by assuming $V$ is continuous. This assumption is very strong. Upper probabilities may not be continuous. In this paper we prove the strong law of large numbers for an upper probability without the continuity assumption whereby random variables are quasi-continuous and the upper probability is generated by a weakly compact family of probabilities on a complete and separable metric sample space.
\end{abstract}

Keywords: Strong Law of Large Numbers; Upper Probability; Weakly Compact; Independence; Quasi-Continuous

\section{Introduction}

Strong law of large numbers under nonadditive probabilities is a much important theory in uncertainty theories and has more applications in statistics, risk measures, asset pricings and many other fields. In 1999 Marinacci [1] first investigated the strong law of large numbers for sequences of independent and identically distributed (IID for short) random variables $\left\{X_{i}\right\}_{i=1}^{\infty}$ relative to a capacity $v$ which is continuous and totally monotone and proved that under regularity condition the limit inferior and limit superior of $\frac{S_{n}}{n}$, where $S_{n}:=\sum_{i=1}^{n} X_{i}$, lie between the two Choquet integrals $\int X_{1} \mathrm{~d} v$ (submean) and $-\int-X_{1} \mathrm{~d} v$ (supermean) induced by this capacity with probability 1 under $v$ (that is, quasi surely), and furthermore, if $v$ is null-additive, then that limit inferior attains the submean and the limit superior attains the supermean quasi surely, respectively. This is different from the law under probability measure $P$ whereby under suitable conditions, such as for IID sequences, $\frac{S_{n}}{n}$ converges to the mathematical expectation of $X_{1}$ almost surely relative to $P$. In 2005 Maccheroni and Marinacci [2] extended the results of Marinacci [1] for a totally monotone capacity on Polish space whereby the bounded variables $X_{i}$ are continuous or simple, or the capacity $v$ is continuous. But the conditions of these two articles on capacity are too strong and not easy to test. And generally capacities can not uniquely determine the (non- linear) expectations relative to the capacities. Motivated by robust statistics and limit theories under sublinear expectations given by Peng in 2007, Chen [3] in 2010 investigated the strong law of large numbers for a pair of lower and upper probabilities $(v, V)$ which are induced by a sublinear expectation $\mathbb{E}$ (see Peng (2012) [4]) whereby the sequence $\left\{X_{i}\right\}_{i=1}^{\infty}$ is IID under $\mathbb{E}$ (the independence is different from classical case and the one in Marinacci [1], see Peng (2012) [4]). He proved if $\mathbb{E}\left[\left|X_{1}\right|^{1+\alpha}\right]<\infty$ for some $\alpha>0$, then those limit inferior and limit superior lie between another submean $-\mathbb{E}\left[-X_{1}\right]$ and supermean $\mathbb{E}\left[X_{1}\right]$ which may not equal the ones given by Choquet integrals (see Chen, $\mathrm{Wu}$ and Li (2012) [5] for details). Furthermore, if we futher assume that $V$ is continuous, then

$$
\begin{aligned}
& V\left(\limsup _{n \rightarrow \infty} \frac{S_{n}}{n}=\mathbb{E}\left[X_{1}\right]\right) \\
= & V\left(\liminf _{n \rightarrow \infty} \frac{S_{n}}{n}=-\mathbb{E}\left[-X_{1}\right]\right)=1
\end{aligned}
$$

$\mathrm{Hu}$ (2012) [6] extends the results of Chen [3] to the sequence of non-identically distributed random variables for the same independence and continuity assumptions. Chen and $\mathrm{Wu}$ (2011) [7] extends Chen [3] to more weaker independence condition without identical distribution assumption, and proves if we further assume that $V$ is continuous, for any subsequence $\left\{n_{i}\right\}_{i=1}^{\infty}$ of $\mathbb{N}$, $\left\{S_{n_{i}}-S_{n_{i-1}}\right\}_{i=1}^{\infty}$ are pairwise weakly independent under $v$, and there exist some constants $\alpha>0, n_{0} \geq 1$, and 
$c_{0}>0$ such that

$$
\sup _{i \geq 1} \hat{E}\left[\left|X_{i}\right|^{1+\alpha}\right]<\infty, \frac{\left|S_{n}\right|}{n} \leq c_{0} \ln (n+1), \forall n \geq 1,
$$

then (1) still holds.

We can see that for the strong law of large numbers (1) under an upper probability, there are two key conditions: well-defined independence and continuity of the upper probability $V$. The continuity assumption of $V$ is based on the second Borel-Cantelli lemma to get $V\left(A_{n}\right.$, i.o. $)=1$ for certain sequence of measurable events $A_{n}$. But as we know in general $V$ is not continuous, since for nonclosed nonincreasing sequence of measurable events $G_{n}$, even if $G_{n} \downarrow G, V\left(G_{n}\right) \downarrow V(G)$ may not hold (see Xu and Zhang (2010) [8] for an example). Hence a natural question is: if $V$ is not continuous, whether does (1) hold? In this paper we will give a confirmative answer. We assume

a1) $\Omega$ is a complete and separable metric space, $F$ is a $\sigma$-algebra of all Borel subsets of $\Omega, P$ is a nonempty subset of $\mathcal{M}$ which is a family of all probabilities on $(\Omega, F)$, and $P$ is also weakly compact;

a2) For each $i \geq 1, \quad X_{i}$ is quasi-continuous, and $\sup _{i \geq 1} \mathbb{E}\left[\left|X_{i}\right|^{2}\right]<\infty$;

a3) $\left\{X_{n}\right\}_{n=1}^{\infty}$ is independent sequence of random variables under $\mathbb{E}$,

where $\mathbb{E}$ is a sublinear expectation corresponding to $P$. In this paper we successfully proved the strong law of large numbers under assumptions a1)-a3) without the continuity assumption of $V$ by transforming that an event $A \in F$ occures with probability 1 under $V$ (that is, $V(A)=1)$ to the problem of its complementary event $A^{c}$, i.e., $v\left(A^{c}\right)=1-V(A)=0$, where $v$ is the conjugate lower probability of $V$ and proving $v\left(B_{n}\right.$, i.o. $)=0$ for some appropriate events $B_{n}$ with $\limsup B_{n} \supset A^{c}$ by using properties of $V$ and $v$.

This paper is organized as follows. In Section 2 we give some basic concepts and useful lemmas. In Section 3 we mainly prove the strong law of large numbers without continuity assumption of upper probability $V$ for IID and continuous sequences. Section 4 extends results of Section 3 and gets the law for non-identically distributed sequence. Section 5 gives an example.

\section{Preliminaries}

Let $\Omega$ be a separable and complete metric space. $F$ is a $\sigma$-algebra of all Borel subsets of $\Omega$. We introduce an upper probability $V$ by

$$
V(A)=\sup _{Q \in P} Q(A), \forall A \in F,
$$

where $P$ is a family of probabilities on $(\Omega, F)$ and weakly compact. Thus its conjugate capacity (see Choquet (1954) [9]), i.e., lower probability is

$$
v(A)=\inf _{Q \in P} Q(A)=1-V\left(A^{c}\right), \forall A \in F,
$$

where $A^{c}$ is the complementary set of $A$. From Huber and Strassen (1973) [10] $V$ and $v$ also satisfy the following properties.

\section{Proposition 1.}

1) $V(\Omega)=v(\Omega)=1, V(\varnothing)=v(\varnothing)=0$.

2) $V(A) \leq V(B), v(A) \leq v(B)$ if $A \subset B, A, B \in F$.

3) $V\left(\sum_{n=1}^{\infty} A_{n}\right) \leq \sum_{n=1}^{\infty} V\left(A_{n}\right), \forall A_{n} \in F, n \geq 1$.

4) $V(A) \geq v(A)$ for all $A \in F$.

5) lower-continuity of $V$ for all sets in $F$ : if $A_{n} \uparrow A, A_{n} \in F$, then $V\left(A_{n}\right) \uparrow V(A)$.

6) upper-continuity of $V$ for all closed sets: if $F_{n} \in F$ closed, $F_{n} \downarrow F$, then $V\left(F_{n}\right) \downarrow V(F)$.

7) lower-continuity of $v$ for all open sets: if $G_{n} \uparrow G, G_{n} \in F$ open, then $v\left(G_{n}\right) \uparrow v(G)$.

8) upper-continuity of $v$ for all sets: if $B_{n} \in F, B_{n} \downarrow B$, then $v\left(B_{n}\right) \downarrow v(B)$.

Now we introduce an upper expectation $\mathbb{E}$ by $V$ in the following

$$
\mathbb{E}[X]=\sup _{Q \in P} E_{Q}[X]
$$

for all $X \in F$ such that $\sup _{Q \in P} E_{Q}[X]<\infty, \quad E_{Q}$ is the linear expectation corresponding to $Q \in P$ such that $E_{Q}\left[I_{A}\right]=Q(A), \forall A \in F$.

Then $\mathbb{E}$ is a sublinear expectation (see Peng [4]) on $(\Omega, H)$, where $H$ is a set of all real-valued random variables $X \in F$ such that $\mathbb{E}[X]<\infty$, that is, $\mathbb{E}$ satisfies that for all $X, Y \in H$,

1) Monotonicity: $\mathbb{E}[X] \leq \mathbb{E}[Y]$ if $X \leq Y$.

2) Constant preserving: $\mathbb{E}[c]=c, \forall c \in \mathbb{R}$.

3) Sub-additivity: $\mathbb{E}[X+Y] \leq \mathbb{E}[X]+\mathbb{E}[Y]$.

4) Positive homogeneity: $\mathbb{E}[\lambda X]=\lambda \mathbb{E}[X], \forall \lambda \geq 0$.

$(\Omega, H, \mathbb{E})$ is called a sublinear expectation space in contrast with probability space. Given

$X=\left(X_{1}, X_{2}, \cdots, X_{n}\right)$, we say $X \in H^{n}$, if $X_{i} \in H$ for all $i=1,2, \cdots, n$. For $X \in H, \quad \mathbb{E}[X]$ is called its supermean, whereas $-\mathbb{E}[-X]$ is called its submean. If $\mathbb{E}[X] \neq-\mathbb{E}[-X]$, then $X$ is said to have mean uncertainty.

In the following we introduce some useful concepts (one can refer to Peng (2010) [4] for details).

Definition 2. An n-dimensional random vector $Y \in H^{n}$ is said to be independent from an m-dimensional random vector $X \in H^{m}$ under $\mathbb{E}$, if for all bounded Lipschitz continuous functions $\varphi \in C_{b, \text { Lip }}\left(\mathbb{R}^{m+n}\right)$, we have 


$$
\mathbb{E}[\varphi(X, Y)]=\mathbb{E}\left[\mathbb{E}[\varphi(x, Y)]_{x=X}\right],
$$

where $\mathbb{E}[\varphi(x, Y)]_{x=X} \in H^{m}$.

Remark 3. In general $Y$ being independent of $X$ under $\mathbb{E}$ does not imply $X$ being independent of $Y$. See Example 3.13 of Chapter I in Peng (2012) [4] as a counterexample.

Definition 4. A sequence $\left\{X_{n}\right\}_{n=1}^{\infty}$ on $(\Omega, H, \mathbb{E})$ is said to be a sequence of independent random variables under $\mathbb{E}$, if for any $i \geq 1, X_{i+1}$ is independent of $\left(X_{1}, X_{2}, \cdots, X_{i}\right)$ under $\mathbb{E}$

Definition 5. A real random variable $X \in F$ is said to be quasi-continuous (q.c. for short) if for any $\varepsilon>0$, there exists an open set $O$ with $V(O)<\varepsilon$ such that $X$ is continuous on $O^{c}$.

Lemma 6. (Denis-Hu-Peng (2011) [11] Theorem 2) For any $A \in F$,

$$
V(A)=\sup \{V(K): \text { compact } K \subset A\} .
$$

Remark 7. Lemma 6 implies that for any $A \in F$,

$$
v(A)=\inf \{v(G) \text { : open } G \supset A\} .
$$

The following Borel-Cantelli lemma is obvious (the readers also can refer to Peng [4] or Chen [3]).

Lemma 8 (Borel-Cantelli Lemma). For any sequence of events $\left\{A_{n}\right\}_{n=1}^{\infty}$ in $F$, if $\sum_{n=1}^{\infty} V\left(A_{n}\right)<\infty$, then

$$
V\left(A_{n}, \text { i.o. }\right)=V\left(\bigcap_{k=1}^{\infty} \bigcup_{n=k}^{\infty} A_{n}\right)=0 .
$$

Lemma 9 (Hu [6] Theorem 3.1). Let $\left\{X_{n}\right\}_{n=1}^{\infty}$ be a sequence of independent random variables on $(\Omega, H, \mathbb{E})$. We assume

1) For any $i \geq 1$, there exist real constants $\underline{\mu}_{i} \leq \bar{\mu}_{i}$, such that $\mathbb{E}\left[X_{i}\right]=\bar{\mu}_{i}$ and $-\mathbb{E}\left[-X_{i}\right]=\underline{\mu}_{i}$;

2) There exist two real constants $\underline{\mu} \leq \bar{\mu}$ such that

$$
\lim _{n \rightarrow \infty} \frac{1}{n} \sum_{i=1}^{n}\left|\underline{\mu}_{i}-\underline{\mu}\right|=0, \lim _{n \rightarrow \infty} \frac{1}{n} \sum_{i=1}^{n}\left|\bar{\mu}_{i}-\bar{\mu}\right|=0 ;
$$

3) $\sup _{n \geq 1} \mathbb{E}\left[X_{n}^{2}\right]<\infty$.

Set $S_{n}=\sum_{i=1}^{n} X_{i}, \forall n \geq 1$. Then for any continuous function $\varphi$ with linear growth on $\mathbb{R}$, we have

$$
\lim _{n \rightarrow \infty} \mathbb{E}\left[\varphi\left(\frac{S_{n}}{n}\right)\right]=\sup _{\underline{\mu} \leq y \leq \bar{\mu}} \varphi(y) .
$$

Lemma 10 (Hu [6] Theorem 3.2 (I)). Let $\left\{X_{n}\right\}_{n=1}^{\infty}$ satisfy all the conditions given in Lemma 9 , then

$$
V\left(\lim _{n \rightarrow \infty} \sup \frac{S_{n}}{n}>\bar{\mu}\right)=V\left(\liminf _{n \rightarrow \infty} \frac{S_{n}}{n}<\underline{\mu}\right)=0 .
$$

\section{Strong Law of Large Numbers}

Theorem 11. Let $\left\{X_{n}\right\}_{n=1}^{\infty}$ be an independent and continuous sequence under $\mathbb{E}$. We assume there exist two real constants $\underline{\mu} \leq \bar{\mu}$ such that $\mathbb{E}\left[X_{i}\right]=\bar{\mu}$, $-\mathbb{E}\left[-X_{i}\right]=\underline{\mu}$ for all $i \geq 1$, and $\sup _{n \geq 1} \mathbb{E}\left[\left|X_{n}\right|^{2}\right]<\infty$. Set $S_{n}=\sum_{i=1}^{n} X_{i}, \forall n \geq 1$. Then

$$
\begin{aligned}
& V\left(\limsup _{n \rightarrow \infty} \frac{S_{n}}{n}=\bar{\mu}\right)=1, \\
& V\left(\liminf _{n \rightarrow \infty} \frac{S_{n}}{n}=\underline{\mu}\right)=1 .
\end{aligned}
$$

Proof. It is obvious that we only need to prove one of the Equations (2) and (3), since on $\Omega$

$$
\limsup _{n \rightarrow \infty} \frac{S_{n}}{n}=-\liminf _{n \rightarrow \infty} \frac{-S_{n}}{n} .
$$

In the following we will prove the Equation (2). It is trivial for $\bar{\mu}=\mu$ and this theorem obviously holds true in this case from (I) of Theorem 1.1 of Chen and $\mathrm{Wu}$ [7] or Lemma 10 . Hence we only need to consider $\bar{\mu}>\mu$ By Lemma 10 or (I) of Theorem 1.1 of Chen and $\mathrm{Wu}$ [7], we have

$$
V\left(\limsup _{n \rightarrow \infty} \frac{S_{n}}{n}>\bar{\mu}\right)=0 .
$$

Hence we only need to prove

$$
V\left(\lim _{n \rightarrow \infty} \sup \frac{S_{n}}{n} \geq \bar{\mu}\right)=1 .
$$

For any subsequence $\left\{n_{k}\right\}_{k=1}^{\infty}$ of $\mathbb{N}$, we denote

$$
\begin{aligned}
& A(a)=\left\{\omega \in \Omega: \lim _{k \rightarrow \infty} \sup \frac{S_{n_{k}}}{n_{k}} \geq a\right\}, \\
& A_{n_{k}}(a)=\left\{\omega \in \Omega: \frac{S_{n_{k}}}{n_{k}} \geq a\right\}, \forall k \geq 1, a \in \mathbb{R} .
\end{aligned}
$$

Since $\left\{X_{n}\right\}_{n=1}^{\infty}$ is a sequence of continuous random variables, thus $A(a)$ and $A_{n_{k}}(a)$ are both closed sets in $F$ for all $a \in \mathbb{R}$ and $k \geq 1$. Thus $A_{n_{k}}^{c}(a)$ is an open set in $F$ for any $k \geq 1$ and $a \in \mathbb{R}$. Then by the upper-continuity of $V$ (see Proposition 1 (6)) for closed sets in $F$, we only need to prove for any fixed constant $\varepsilon \in(0, \bar{\mu}-\mu)$,

$$
V\left(\lim _{n \rightarrow \infty} \sup \frac{S_{n}}{n} \geq \bar{\mu}-\varepsilon\right)=1 .
$$

Equivalently, we only need to prove for any fixed $\varepsilon \in(0, \bar{\mu}-\underline{\mu})$,

$$
v\left(\lim _{n \rightarrow \infty} \sup \frac{S_{n}}{n}<\bar{\mu}-\varepsilon\right)=0 .
$$


Then it is sufficient to find an increasing subsequence $\left\{n_{k}\right\}_{k=1}^{\infty}$ of $\mathbb{N}$ such that for any fixed $\varepsilon \in(0, \bar{\mu}-\underline{\mu})$,

$$
v\left(\lim _{k \rightarrow \infty} \sup \frac{S_{n_{k}}}{n_{k}}<\bar{\mu}-\varepsilon\right)=0 .
$$

Noticing that

$$
\begin{aligned}
& v\left(\lim _{k \rightarrow \infty} \sup \frac{S_{n_{k}}}{n_{k}}<\bar{\mu}-\varepsilon\right) \\
\leq & v\left(\liminf _{k \rightarrow \infty} \frac{S_{n_{k}}}{n_{k}}<\bar{\mu}-\varepsilon\right) \\
= & v\left(\bigcap_{m=1}^{\infty} \bigcup_{k=m}^{\infty} A_{n_{k}}^{c}(\bar{\mu}-\varepsilon)\right) \\
= & \lim _{m \rightarrow \infty} v\left(\bigcup_{k=m}^{\infty} A_{n_{k}}^{c}(\bar{\mu}-\varepsilon)\right) \\
= & \lim _{m \rightarrow \infty}\left[1-V\left(\bigcap_{k=m}^{\infty} A_{n_{k}}(\bar{\mu}-\varepsilon)\right)\right] \\
= & 1-\lim _{m \rightarrow \infty} V\left(\bigcap_{k=m}^{\infty} A_{n_{k}}(\bar{\mu}-\varepsilon)\right) \\
= & 1-\lim _{m \rightarrow \infty} \lim _{l \rightarrow \infty} V\left(\bigcap_{k=m}^{l} A_{n_{k}}(\bar{\mu}-\varepsilon)\right),
\end{aligned}
$$

since $A_{n_{k}}(\bar{\mu}-\varepsilon)$ are all closed sets and $V$ is uppercontinuous for closed sets.

In addition, for any $m \geq 2, l \geq m$,

$$
\begin{aligned}
& V\left(\bigcap_{k=m}^{l} A_{n_{k}}(\bar{\mu}-\varepsilon)\right) \\
\geq & V\left(\bigcap_{k=m}^{l}\left[\left\{Y_{k} \geq \bar{\mu}-\varepsilon\right\} \cap\left\{\frac{S_{n_{k-1}}}{n_{k-1}} \geq \bar{\mu}-\varepsilon\right\}\right]\right) \\
= & V\left(\bigcap_{k=m}^{l}\left\{Y_{k} \geq \bar{\mu}-\varepsilon\right\} \cap\left\{\frac{S_{n_{m-1}}}{n_{m-1}} \geq \bar{\mu}-\varepsilon\right\}\right) \\
\geq & \mathbb{E}\left[\prod_{k=m}^{l} g^{\delta}\left(Y_{k}\right) g^{\delta}\left(\frac{S_{n_{m-1}}}{n_{m-1}}\right)\right],
\end{aligned}
$$

where $Y_{k}=\frac{S_{n_{k}}-S_{n_{k-1}}}{n_{k}-n_{k-1}}$ and

$$
g^{\delta}(x)=\left\{\begin{array}{l}
1, \quad x \geq \bar{\mu}-\varepsilon+\delta \\
\frac{1}{\delta}(x-\bar{\mu}+\varepsilon), \quad \bar{\mu}-\varepsilon<x<\bar{\mu}-\varepsilon+\delta ; \\
0, \quad x \leq \bar{\mu}-\varepsilon,
\end{array}\right.
$$

where $\delta$ is any fixed constant in $(0, \varepsilon)$. It is obvious that for any fixed $\delta \in(0, \varepsilon), \quad g^{\delta}$ is a bounded and Lipschitz continuous function on $\mathbb{R}$. Thus by the independence assumption we know that

$$
\begin{aligned}
& V\left(\bigcap_{k=m}^{l} A_{n_{k}}(\bar{\mu}-\varepsilon)\right) \\
\geq & \prod_{k=m}^{l} \mathbb{E}\left[g^{\delta}\left(\frac{S_{n_{k}}-S_{n_{k-1}}}{n_{k}-n_{k-1}}\right)\right] \mathbb{E}\left[g^{\delta}\left(\frac{S_{n_{m-1}}}{n_{m-1}}\right)\right]
\end{aligned}
$$

And then by Lemma 9 for any fixed $m \geq 2$, and $l \geq m$, if we choose a small constant $\tau=\frac{1}{(l+1-m) m}$, then there exists an integer $N=N(\delta, \varepsilon, l, m) \geq 1$, such that for any $n_{k}-n_{k-1} \geq N$, we have

$$
\begin{aligned}
& \mathbb{E}\left[g^{\delta}\left(\frac{\bar{S}_{n_{k}-n_{k-1}}}{n_{k}-n_{k-1}}\right)\right] \geq \sup _{\underline{\mu} \leq y \leq \bar{\mu}} g^{\delta}(y)-\tau=1-\tau, \\
& m \leq k \leq l,
\end{aligned}
$$

where we denote $\bar{S}_{n_{k}-n_{k-1}}:=S_{n_{k}}-S_{n_{k-1}}$ for all fixed $k$ with $m \leq k \leq l$.

Taking $n_{k}=N^{k}$ for any $m \leq k \leq l$, we can obtain

$$
V\left(\bigcap_{k=m}^{l} A_{n_{k}}(\bar{\mu}-\varepsilon)\right) \geq(1-\tau)^{l+1-m} \mathbb{E}\left[g^{\delta}\left(\frac{S_{n_{m-1}}}{n_{m-1}}\right)\right] \text {. }
$$

Then letting $l$ tend to $\infty$ and then letting $m$ tend to $\infty$ on both sides of inequality (7), by Lemma 9 again, we can get

$$
\begin{aligned}
& \lim _{m \rightarrow \infty} \lim _{l \rightarrow \infty} V\left(\bigcap_{k=m}^{l} A_{n_{k}}(\bar{\mu}-\varepsilon)\right) \\
\geq & \lim _{m \rightarrow \infty} \lim _{\tau \rightarrow 0^{+}}(1-\tau)^{\frac{1}{\tau m}} \mathbb{E}\left[g^{\delta}\left(\frac{S_{n_{m-1}}}{n_{m-1}}\right)\right] \\
= & \lim _{m \rightarrow \infty} \mathbb{E}\left[g^{\delta}\left(\frac{S_{n_{m-1}}}{n_{m-1}}\right)\right] \lim _{m \rightarrow \infty} \mathrm{e}^{-\frac{1}{m}}=1
\end{aligned}
$$

Thus from (5) and (8) we can obtain

$$
v\left(\lim _{k \rightarrow \infty} \sup \frac{S_{n_{k}}}{n_{k}}<\bar{\mu}-\varepsilon\right) \leq 0 .
$$

Therefore, (4) holds true. We complete the whole proof. $\square$

Remark 12. If $\underline{\mu}=\bar{\mu}=: \mu$, then from the Theorem 11 we can see that $V\left(\lim _{n \rightarrow \infty} \frac{S_{n}}{n}=\mu\right)=1$. This is just a trivial case for sequences without mean uncertainty.

Corollary 13. Let $\left\{X_{n}\right\}_{n=1}^{\infty}$ be a sequence of quasicontinuous random variables and satisfy all other conditions except for the continuity given in Theorem 11, then Theorem 11 still holds.

Proof. Similarly as the arguments in the proof of Theorem 11, we only need to prove

$$
V\left(\limsup _{n \rightarrow \infty} \frac{S_{n}}{n} \geq \bar{\mu}\right)=1,
$$

when $\bar{\mu}>\mu$.

By the assumptions we know that for each $n \geq 1$ and any constant $2^{-n}>0$, there exists an open subset $O_{n}$ of $\Omega$ with $V\left(O_{n}\right)<2^{-n}$, such that $X_{n}$ is continuous on $O_{n}^{c}$. Denote $O=\bigcap_{n=1}^{\infty} \bigcup_{k=n}^{\infty} O_{k}$, then by Borel-Can- 
telli lemma (see Lemma 8) we can obtain

$$
v(O)=V(O)=0 \text {. }
$$

For any $1>\delta>0$, there exist an increasing subsequence $\left\{n_{k}\right\}_{k=1}^{\infty}$ of $\mathbb{N}$, an integer $N=N_{\delta} \geq 1$ and an open set $G^{\delta} \supset O$ (by Remark 7) satisfying

$$
v\left(G^{\delta}\right) \leq v(O)+\delta=\delta
$$

such that when $n_{1} \geq N$, we have

$$
G^{\delta} \supset \bigcup_{k=1}^{\infty} O_{n_{k}} \text {. }
$$

Then $X_{n_{k}}$ is continuous on $\left(G^{\delta}\right)^{c}$. By Lemma 6 , for any $\varepsilon>0$, we can find a compact set $K^{\varepsilon} \subset\left(G^{\delta}\right)^{c}$ with $a_{\varepsilon}=V\left(K^{\varepsilon}\right) \neq 0$ such that

$$
a_{\varepsilon}=V\left(K^{\varepsilon}\right) \geq V\left(\left(G^{\delta}\right)^{c}\right)-\varepsilon \geq 1-\delta-\varepsilon .
$$

Then we have

$$
\begin{aligned}
& V\left(\limsup _{n \rightarrow \infty} \frac{S_{n}}{n} \geq \bar{\mu}\right) \\
\geq & V\left(\limsup _{k \rightarrow \infty} \frac{S_{n_{k}}}{n_{k}} I_{\left(G^{\delta}\right)^{c}} \geq \bar{\mu}\right) \\
\geq & \left(V \lim _{k \rightarrow \infty} \sup \frac{S_{n_{k}}}{n_{k}} I_{K^{\varepsilon}} \geq \bar{\mu}\right), \forall \varepsilon>0 .
\end{aligned}
$$

For $\varepsilon>0$, we define

$$
c_{V}^{\varepsilon}(A)=\frac{1}{a_{\varepsilon}} V(A), \forall A \in K^{\varepsilon} \bigcap F=: F^{\varepsilon} .
$$

Then it is obvious that $c_{V}^{\varepsilon}$ is a capacity on $K^{\varepsilon}$ and satisfies all the properties of $V$ given in Proposition 1 where $\Omega$ is substituted by $K^{\varepsilon}$. We also denote by $H^{\varepsilon}$ the set of all random variables $X \in F^{\varepsilon}$ such that $\mathbb{E}[X]<\infty$. Thus on $\left(K^{\varepsilon}, H^{\varepsilon}, \mathbb{E}\right),\left\{X_{n_{k}} I_{K^{\varepsilon}}\right\}_{k=1}^{\infty}$ is an independent and continuous sequence. Since $K^{\varepsilon}$ is also a complete and separable metric space, by Theorem 11 we have

$$
c_{V}^{\varepsilon}\left(\lim _{k \rightarrow \infty} \sup \frac{S_{n_{k}}}{n_{k}} I_{K^{\varepsilon}} \geq \bar{\mu}\right)=1 .
$$

Then from (10)-(12) we have

$$
V\left(\limsup _{n \rightarrow \infty} \frac{S_{n}}{n} \geq \bar{\mu}\right) \geq a_{\varepsilon} \geq 1-\delta-\varepsilon .
$$

Letting $\varepsilon$ and $\delta$ tend to 0 in inequality (13) we can derive

$$
V\left(\limsup _{n \rightarrow \infty} \frac{S_{n}}{n} \geq \bar{\mu}\right) \geq 1,
$$

which implies (9). We complete the whole proof of this corollary.

\section{Extensions}

In Section 3 we get that the submean $\mu$ and the supermean $\bar{\mu}$ are the inferior and superior limits of the arithmetic average of the first $n$ random variables $X_{1}, X_{2}, \cdots, X_{n}$ given in Theorem 11 , respectively, with probability 1 under the upper probability $V$. In fact, except the two values, any other value $x \in(\underline{\mu}, \bar{\mu})$ is still the limit of some subsequence of $\left\{X_{n}\right\}_{n=1}^{\infty}$, with probability 1 under $V$. We can see it in the following theorem.

Theorem 14. Under assumptions of Theorem 11, we have for any $x \in[\underline{\mu}, \bar{\mu}]$

$$
V\left(x \in C\left(\frac{S_{n}}{n}\right)\right)=1,
$$

where $C\left(y_{n}\right)$ is a cluster of limit points of a real sequence $\left\{y_{n}\right\}_{n=1}^{\infty}$.

Proof. For $x=\mu$ and $x=\bar{\mu}$, the result has been obtained in Theore $\bar{m} 11$. For the trivial case, $\mu=\bar{\mu}$, it is obvious. Now we consider $\bar{\mu}>\underline{\mu}$ and any $x \in(\underline{\mu}, \bar{\mu})$. We can notice that

$$
\begin{aligned}
V\left(x \in C\left(\frac{S_{n}}{n}\right)\right) & =V\left(\liminf _{n \rightarrow \infty}\left|\frac{S_{n}}{n}-x\right|=0\right) \\
& =\lim _{\varepsilon \rightarrow 0} V\left(\liminf _{n \rightarrow \infty}\left|\frac{S_{n}}{n}-x\right| \leq \varepsilon\right),
\end{aligned}
$$

where $\varepsilon$ is any constant in $(0, \min \{\bar{\mu}-x, x-\underline{\mu}\})$, since $V$ is upper-continuous for closed sets. Thus we only need to find an increasing subsequence $\left\{n_{k}\right\}_{n=1}^{\infty}$ of $\mathbb{N}$ such that for any $\varepsilon \in(0, \min \{\bar{\mu}-x, x-\underline{\mu}\})$, we have

$$
v\left(\lim _{n \rightarrow \infty} \sup \left|\frac{S_{n}}{n}-x\right|>\varepsilon\right)=0,
$$

Following the arguments in the proof of Theorem 11 we can obtain

$$
\begin{aligned}
& v\left(\limsup _{n \rightarrow \infty}\left|\frac{S_{n}}{n}-x\right|>\varepsilon\right) \\
& \leq 1-\lim _{m \rightarrow \infty} \lim _{l \rightarrow \infty} V\left(\bigcap_{k=m}^{l}\left\{\left|Y_{k, x}\right| \leq \varepsilon\right\} \cap\left\{\left|Z_{k, x}\right| \leq \varepsilon\right\}\right) \\
& \leq 1-\lim _{m \rightarrow \infty} \lim _{l \rightarrow \infty} \prod_{k=m}^{l} \mathbb{E}\left[f^{\delta}\left(Y_{k, x}\right)\right] \mathbb{E}\left[f^{\delta}\left(Z_{k, x}\right)\right],
\end{aligned}
$$

where $Y_{k, x}=\frac{S_{n_{k}}-S_{n_{k-1}}}{n_{k}-n_{k-1}}-x$ and $Z_{k, x}=\frac{S_{n_{m-1}}}{n_{m-1}}-x$ for 
any $k \geq 1$ and $x \in(\underline{\mu}, \bar{\mu}), \delta$ is any given constant in $(0, \varepsilon)$ and

$$
f^{\delta}(y)=\left\{\begin{array}{l}
0, \quad y \geq \varepsilon ; \\
\frac{1}{\delta}(\varepsilon-y), \quad \varepsilon-\delta<y<\varepsilon ; \\
1, \quad-\varepsilon+\delta \leq y \leq \varepsilon-\delta ; \\
\frac{1}{\delta}(y+\varepsilon), \quad-\varepsilon<y<-\varepsilon+\delta ; \\
0, \quad y \leq-\varepsilon .
\end{array}\right.
$$

Then by using the same arguments as in the proof of Theorem 11 we also can prove that (14) holds true. The whole proof is complete. $\square$

The following corollary is obvious.

Corollary 15. Under the conditions of Theorem 11, for any continuous real function $\varphi$ on $\mathbb{R}$, we have for all $x \in[\underline{\mu}, \bar{\mu}]$,

$$
V\left(\varphi(x) \in C\left(\varphi\left(\frac{S_{n}}{n}\right)\right)\right)=1 .
$$

In particular,

$$
\begin{aligned}
& V\left(\lim _{n \rightarrow \infty} \sup \varphi\left(\frac{S_{n}}{n}\right)=\sup _{y \in[\underline{\mu}, \bar{\mu}]} \varphi(y)\right)=1, \\
& V\left(\liminf _{n \rightarrow \infty} \varphi\left(\frac{S_{n}}{n}\right)=\inf _{y \in[\underline{\mu}, \bar{\mu}]} \varphi(y)\right)=1, \forall \varphi \in C(\mathbb{R}) .
\end{aligned}
$$

We also can extend Theorem 11, Theorem 14 and Corollary 15 to the sequences with different submeans and supermeans as follows.

Theorem 16. Let $\left\{X_{n}\right\}_{n=1}^{\infty}$ be an independent and continuous sequence under $\mathbb{E}$ and satisfy conditions (1)-(3) of Lemma 9. Set $S_{n}=\sum_{i=1}^{n} X_{i}, \forall n \geq 1$. Then for any $\varphi \in C(\mathbb{R})$ we have

$$
V\left(\varphi(x) \in C\left(\varphi\left(\frac{S_{n}}{n}\right)\right)\right)=1, \forall x \in[\underline{\mu}, \bar{\mu}] .
$$

Proof. By Lemma 10 and the proofs of Theorem 11 and Theorem 14 we only need to check whether (6) and (8) hold true under our assumptions of this theorem. In fact, from Lemma 9 they are obviously satisfied. Hence this theorem holds. $\square$

From the proof of Corollary 13 and Theorem 16 we can immediately obtain the following corollary.

Corollary 17. Theorem 16 still holds when continuity assumption is substituted by quasi-continuity condition and condition (2) of Lemma 9 is replaced by the following condition:

(2') there exist real constants $\underline{\mu} \leq \bar{\mu}$ such that

$$
\lim _{n \rightarrow \infty} \bar{\mu}_{n}=\bar{\mu}, \lim _{n \rightarrow \infty} \underline{\mu}_{n}=\underline{\mu} \text {. }
$$

\section{An Example}

Let $\Omega=C[0, T]$ with the supremum norm. Then $\Omega$ is a Banach space and compact, thus it is a separable and complete metric space with the distance generated by the norm of the space. Then we can define a $G$-expectation $E_{G}$, a special sublinear expectation (see Peng [4] for details), where $G(a)=\frac{1}{2}\left(a^{+}-\sigma^{2} a^{-}\right), \quad \sigma$ is a nonnegative real number less than 1 . Then for any bounded and independent sequence $\left\{X_{n}\right\}_{n=1}^{\infty}$ with the same submean $\mu$ and supermean $\bar{\mu}$ in $L_{G}^{1}\left(F_{T}\right)$ under $E_{G}$, by Theorem 11 we have

$$
V\left(\lim _{n \rightarrow \infty} \sup \frac{1}{n} \sum_{i=1}^{n} X_{i}=\bar{\mu}\right)=V\left(\liminf _{n \rightarrow \infty} \frac{1}{n} \sum_{i=1}^{n} X_{i}=\underline{\mu}\right)=1,
$$

where $V$ is generated by $E_{G}$, since this sequence is a sequence of quasi-continuous random variables and from Denis, $\mathrm{Hu}$ and Peng [11] $E_{G}$ can be represented as supremum of a family of linear expectations corresponding to a family of probabilities which is weakly compact.

\section{Acknowledgements}

This research is supported by WCU (World Class University) program through the National Research Foundation of Korea funded by the Ministry of Education, Science and Technology (R31-20007). The author gratefully thanks the referees for their careful reading.

\section{REFERENCES}

[1] M. Marinacci, "Limit Laws of Non-Additive Probabilities and Their Frequentist Interpretation," Journal of Economic Theory, Vol. 84, No. 2, 1999, pp. 145-195. doi:10.1006/jeth.1998.2479

[2] F. Maccheroni and M. Marinacci, "A Strong Law of Large Numbers for Capacities," Annals of Probability, Vol. 33, No. 3, 2005, pp. 1171-1178. doi: $10.1214 / 009117904000001062$

[3] Z. Chen, "Strong Laws of Large Numbers for Capacities," Unpublished, 2010. http://arxiv.org/abs/1006.0749

[4] S. Peng, "Nonlinear Expectations and Stochastic Calculus under Uncertainty-with Robust Central Limit Theorem and G-Brownian Motion," Unpublished, 2012. http://arxiv.org/abs/1002.4546

[5] Z. Chen, P. Wu and B. Li, "A Strong Law of Large Numbers for Non-additive Probabilities," International Journal of Approximate Reasoning, 2012. http://www.sciencedirect.com/science/article/pii/S088861 3X12000783

[6] F. Hu, "General Laws of Large Numbers under Sublinear Expectations," 2012. http://arxiv.org/abs/1104.5296

[7] Z. Chen and P. Wu, "Strong Laws of Large Numbers for Bernoulli Experiments under Ambiguity," Nonlinear Ma- 
thematics for Uncertainty and Its Applications, Advances in Intelligent and Soft Computing, Vol. 100, 2011, pp. 1930.

http://link.springer.com/chapter/10.1007\%2F978-3-642-2 2833-9 2? LI=true

[8] J. Xu and B. Zhang, "Martingale Property and Capacity under G-Framework," Electronic Journal of Probability, Vol. 15, No. 67, 2010, pp. 2041-2068.

[9] G. Choquet, "Theory of Capacities," Annales de Institute Fourier, Vol. 5, 1954, pp. 131-295.
[10] P. J. Huber and V. Strassen, "Minimax Tests and the Neyman-Pearson Lemma for Capacities," The Annals of Statistics, Vol. 1. No. 2, 1973, pp. 251-263. doi:10.1214/aos/1176342363

[11] L. Denis, M. Hu and S. Peng, "Function Spaces and Capacity Related to a Sublinear Expectation: Application to G-Brownian Motion Paths," Potential Analysis, Vol. 34, No. 2, 2011, pp. 139-161. doi:10.1007/s11118-010-9185-x 